\title{
A Review on Nanoporous Metals
}

\author{
Toyin Jibowu* \\ Faculty of Engineering, Chemical and Biochemical Engineering, University of Western Ontario,Canada
}

\begin{abstract}
Porous metals are materials that have excellent functional and structural properties. They are lightweight materials with properties such as low bulk density, high surface area, low thermal conductivity, good penetrability, energy management use, noise attenuation, vibration suppression etc.

Nanomaterials are a subset of porous materials which have large porosities of $0.4 \mathrm{~nm}$ and above and have pore diameters of 1-100nm. Nanoporous metals have high surface to volume ratio, with a high surface area and large porosity and ordered uniform pore structure.

The applications that nanoporous materials can be used for are catalysis, separation and sensing, drug delivery, etc. They are used for sensing glucose in a diabetics blood, catalysis of oil into hydrocarbons, encapsulating drugs and allowing for a slow controlled release of the drug and many more applications that have the ability to revolutionize the way medication, sensors and catalysis due to their unique properties.
\end{abstract}

\section{Introduction}

\section{Porous metals}

Porous metals are a new class of engineering materials that have excellent functional and structural properties. They are lightweight materials that have the characteristics of metals but also have useful characteristics such as low bulk density, high surface area, low thermal conductivity, good penetrability, energy management use, noise attenuation, vibration suppression etc.

These materials have a wide range of applications and have emerged as a focus of great attention in the material science and material engineering field.

The presence of pores in a material allows the material to have new properties that the bulk material does not have. Porous materials have porosity between 0.2 to 0.95 (volume ratio of pore space to the total volume of material). Pores are classified in two types open pores which connect to the surface material and closed pores which are isolated from the outside. In application such as adsorption, catalysis and sensing one used open pores. In separation, catalysis, filtration or membranes penetrating open pore are required. Closed pore materials are used in thermal insulation or lightweight structural applications. Pores can have various shapes and morphology such as cylindrical, spherical and slit types.

Pore size is classified by the International Union of Pure and Applied Chemistry:

Micropores are pores smaller than $2 \mathrm{~nm}$ in diameter, mesopores are pores between 2 to $50 \mathrm{~nm}$ and macropres are larger than $50 \mathrm{~nm}$.

\section{Properties and characterization of Nanoporous materials}

Nanomaterials are a subset of porous materials which have large porosities greater than $0.4 \mathrm{~nm}$ and pore diameters of $1-100 \mathrm{~nm}$. In the field of chemical functional porous materials the term nanoporous refers to porous materials having diameters between $1-100 \mathrm{~nm}$. Nanoporous materials have high surface to volume ratio, with a high surface area and large porosity and ordered uniform pore structure. They can be used for many applications such as catalysis, separation and sensing. Inorganic nanoporous materials made of oxide are non toxic, inert and chemically and thermally stable.

A unique type of porous metals are nanoporous metals which have a high surface to volume ratio, high surface area, large porosity and uniform pore structure. They have functional applications such as catalysis, sensing and separation.

Nanoporous materials have a unique set of properties that bulk materials do not have such as high specific area, shape selective effects, fluid permeability. Different nanoporous materials have varying pore size, porosity, pore size distribution and composition and surface properties as you can see in Table 1 [1].

\section{Methadology}

\section{Nanoporous Metal Preparation}

Porous materials are fabricated in several different ways.The two most common ways to make porous materials are using the Hard Template Method and Soft Template Method.

\section{Hard Template Method}

Closed packed colloidal crystals are effective templates for preparing porous metals. Most common spheres used in these templates are silica, polymer spheres in the nanometer to micrometer size range. These spheres can be self-assembled on solid substrates to form uniformly arranged colloidal crystals in three dimensions. During electrodeposition metal ions from an electrolyte solution are reduced and fill the interstices of the colloidal crystal templates through

Correspondence to: Toyin Jibowu, Faculty of Engineering, Chemical and Biochemical Engineering, University of Western Ontario, Canada, Tel: 519-6612131; E-mail: ToyinJibowu@gmail.com

Received: July 03, 2016; Accepted: July 18, 2016; Published: July 22, 2016 
manipulation of the deposition variables such as voltage or current density, ph value and concentration of solution. Electrodeposition is only for conductive surfaces and is not for insulating surfaces. Chemical reduction of metal ions with a reducing agent allows the preparation of porous metals without using a conductive substrate. Several methods are used to remove colloidal crystal templates. Silica spheres are removed by immersion in dilute hydrofluoric acid whereas polystyrene spheres are dissolved with other solvents such as trichloromethane, which can be seen in Figure 1 [2]. The size of the small pores and the surface area of the porous metals can be manipulated by tailoring the sizes of colloidal spheres.

Periodic arrays of hollow metal shells can be created, which is shown in Figure 2 [2]. This can be done by post synthesized core shell spheres with a thin layer of metal are able to be arranged on a substrate through filtration, sedimentation or solvent evaporation. The porosity is created by removing the colloidal sphere cores by heat treatment and dissolution.

\section{Soft Template Method}

Lytropic liquid crystalline phases of amphiphilic molecules such as block copolymers contain long range periodic nanostructures with lattice parameters in the tens of nanometers. Depending on the surfactant concentration the structure of the LLC is changed from a micellar solution to $\left(\mathrm{L}_{\mathrm{I}}\right)$ through micellar cubic $\left(\mathrm{I}_{\mathrm{I}}\right)$, hexagonal $\left(\mathrm{H}_{\mathrm{I}}\right)$, bicontinuous cubic $\left(\mathrm{V}_{\mathrm{I}}\right)$, lamellar $\left(\mathrm{L}_{\alpha}\right)$, to inverse micellar $\left(\mathrm{L}_{2}\right)$ phases.

Thus the LLC template method is a versatile way to create mesoporous metals since various pore structures can be created by changing composition of reaction baths. Metals precursors are reduced in the LLC phase leads to formation of mesoporous metals that have well defined and long ranged porous nanostructure. The LLC template is then removed. Pore size of these mesoprous films are determined by the length of the alkyl chain surfactant used while the formation of stable mesophases of LLC in the presence of metal ions is necessary to create continuous metal films, which can be seen in Figure 3. The pore size detected were from 1.7 to $10 \mathrm{~nm}$.

\section{Applications of Nanoporous Metals}

\section{Environmental separations}

As the regulatory limits on environmental emissions become

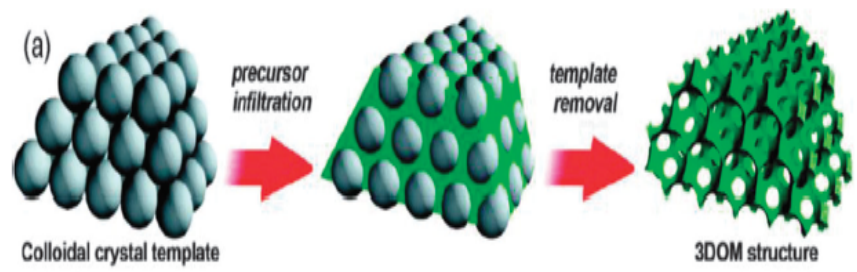

Figure 1. Creation of Nanoporous materials using hard template method.
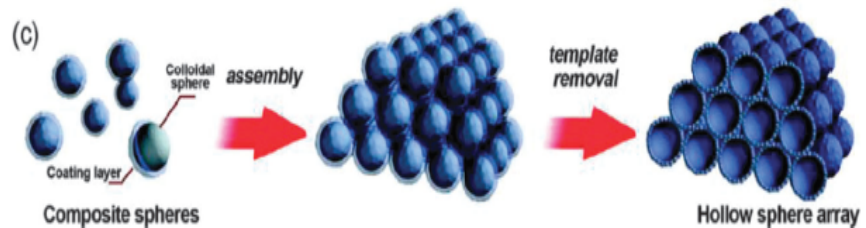

Figure 2. Creation of nanoporous materials using hard template method: composite spheres.

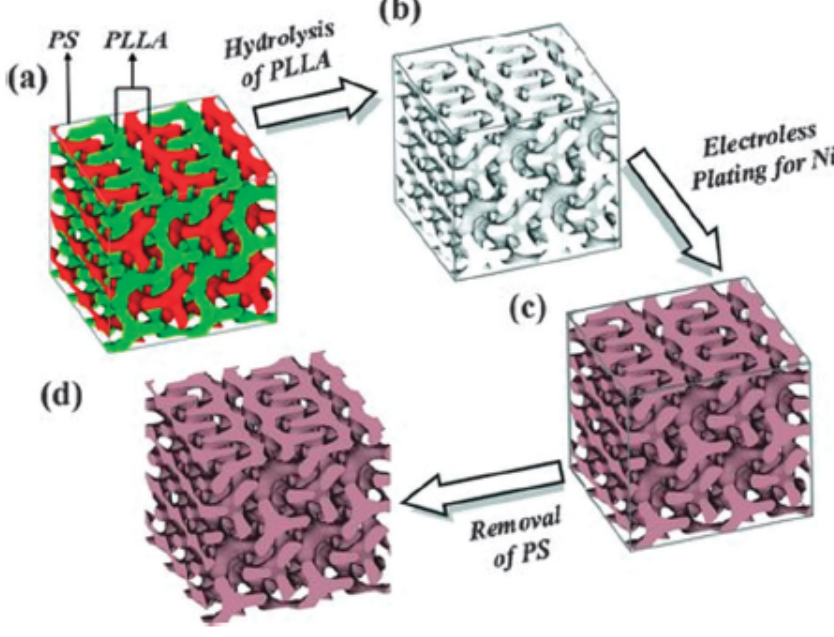

Figure 3. Creation of nanoporous materials using soft template method.

more strict industries have developed separation technologies that remove contaminants and pollutants from waste gas and water streams. Adsorption processes and membrane separations are the two dominant technologies. Adsorbent materials and membranes are being applied and new adsorbent membranes are invented for environmental applications that remove sodium oxide nitrogen oxide and VOCs emissions. Adsorbents of traditional types such as carbons, zeolites, silica gel and activated alumina have a market estimate in the billions of US dollar per year. Some adsorbent materials and their applications are listed in the table below (Table 1).

\section{Clean Energy Production and storage}

Future energy supply will be dependent on hydrogen as a clean energy carrier. Hydrogen can be produced from fossil fuels, water electrolysis and biomass. However hydrogen must be produced cleanly. Key to the process of cost effective conversion of coal to hydrogen and carbon capture is nanoporous material catalysts. Fuel cell development will also be important in which hydrogen will be the dominant fuel and converted into electricity, leaving water as a byproduct. Nanoporous material will be important in this process, nanomaterials such as carbon nanotubes show great promise of being the future catalysts in these fuel cells.

\section{Catalysis}

Heterogeneous catalysis has had a major impact on chemical and fuel production. More efficient catalytic processes are required to improve catalytic activity and selectivity. Therefore a tailor design of catalytic materials with desired microstructures are needed. Nanoporous materials will be used because of their large and accessible surface area of the catalyst. Nanoporous metals have high surface areas, low specific densities and rich surface chemistry and are highly efficient electro catalysts for the oxidation of small organic molecules e.g. methanol, ethanol and formic acid. Platinum is considered to be the best catalysts among pure metals for these reactions. The mass specific activity is comparable to platinum catalysts although only one quarter of the pore surface area is electrochemically accessible. The roughness factor is an important factor for determining catalytic performance, which is the electrochemical active surface area to geometric area. There is a longer residence time of reactants in nanoporous environment which allows the reactant molecules to stay longer inside the pores 
Table 1. Classification of Nano porous materials.

\begin{tabular}{|l|c|c|c|c|c|}
\hline \multicolumn{1}{|c|}{ Polymeric } & Carbon & Glass & Alumino-silicate & Oxides \\
\hline Pore Size & Meso-Macro & Micro-meso & Meso-macro & Micro-meso & Micro-meso \\
\hline Surface area/Porosity & Low $>0.6$ & High $0.3-0.6$ & Low 0.3-0.6 & High $0.3-0.7$ & Medium $0.3-0.6$ \\
\hline Permeability & Low-medium & Low-medium & High & Low $0.1-0.7$ \\
\hline Strength & Medium & Low & Strong & Wew-medium & Weak \\
\hline Thermal stability & Low & High & Good & Medium-high & Medium-high \\
\hline Chemical stability & Low-medium & High & High & High & Very High \\
\hline Costs & Low & High & High & Low-medium & Medium \\
\hline Life & Short & Long & Long & Medium-Long & Medium \\
\hline
\end{tabular}

and have more opportunities to collide with the electrode surface than those of nonporous electrodes [3].

\section{Sensors and actuators}

Nanoparticles and nanoporous materials possess large surface areas and high sensitivity to slight changes in environment. These materials are widely used as sensor and actuator materials. Gas sensors sensitivity is dependent on surface areas and gas sensors based on nanoporous metals such as titanium oxide or zinc oxide are being developed and applied to detectors of combustible gases. Gas sensors rely on the detection of electric resistivity change upon change in gas concentration and their sensitivity is dependent on surface area.

\section{Biological applications}

Nanomaterials are assembled and structured on the nanometer scale and are attractive for biotechnology applications because they can be used for their material topography and spatial distribution of functional groups to control proteins, cells and tissue interactions and for bioseperation.

Nanoporous materials are often bio-compatible afford the capability to build enzymatic nanomaterials that mimic biological reactions. Immobilizing enzymes onto nanoporous materials can be used for biological reactors to produce drugs, decontaminate waste etc.

Nanoporous material can be used for biosensors. Piezoelectric biosensors utilizing high surface area nanoporous coatings have increased sensitivity in detection. Immobilized biological molecules on the surface of nanoporous silica can be used as a biological detection system and the piezo electrical cantilevers can serve as a transducer there is a shift in resonance frequency of the cantilever when molecules absorb on the surface. The incorporation of nanoporous silica between transducer and biological detection system increases surface to volume ratio of cantilever increases sensitivity of resonant frequency of oscillator to changes in mass.

\section{Cell Culture}

Porous silicon has revealed to be convenient cell culture platforms for the growth of cells such as mammalian cells, neuronal cells, lens cells etc.

Substrate topography affects cell functions such as adhesion, proliferation, migration and differentiation. The controllable and tunable pore size and structure of silicon allows for substrates for the proliferation and adhesion of cells.

Increased cell adhesion and growth is improved by surface modification such as introducing functional groups. Amine terminated modifications are widely used for porous silicon functionalization because they improve silicon stability and enhance cell adhesion.

\section{Drug Delivery}

Porous silicon is suitable material for drug delivery due to its high porosity and high surface area. Therapeutic agents have been loaded into porous silicon pores such as small molecules such as doxorubicin. For drug delivery applications several aspects must be considered such as the pore size since the molecule must be smaller than a pore diameter to penetrate into the pores. Porosity is important since the quantity of the drug loaded into the pores is related to capacity of pores.

\section{Nanoparticles}

The porous silicon most widely used for biological applications is nanoparticles. Porous silicon nanoparticles have the same interesting properties for biological applications as the material they are made of e.g. biocompatibility, tunable porous structure etc.

The advantages of the nanometric size allows it to be used for localized treatments they can freely circulate the bloodstream and be phagocytosed by cells. In cancer therapy porous silicon particles are suitable for minimally invasive to the body.

\section{Sensors}

The optical and electrical properties of porous silicon are key parameters used in many chemical and biological sensing applications. Most porous silicon based sensors are interferometric sensors where the optical response of the porous silicon structure is altered with the introduction of the element to be detected in the pores. With the introduction of the molecule to be detected inside the pores the effective refractive index of the porous layers change leading to a shift in reflectance peaks. These sensors have been used to detect organic vapours, glucose, etc.

\section{Templates}

Using porous silicon as a template provides the means for construction of complex structures. Porous silicon is an appropriate template because it can be fabricated with high precision and uniformity and porosity, pore size and pore depth can be tuned by adjusting the electrochemical preparation techniques. Fibers, tubes or porous layers of several materials are fabricated by replicating the porous silicon structure. This template assisted technique has advantage of readily create large area and ordered arrays of micro and nanostructures with control over structural parameter

\section{Conclusion}

In conclusion, nanoporous metals are an important material that has applications in many areas including biomedical, catalysis and green energy Nanoporous metals have high surface area, tunable pore 
sizes, and high surface to volume ratio and can be functionalized.

These nanoporous metals have been used to created drugs for cancer, catalysis of reactions due to its high surface area, capturing hydrogen and carbon into its pores and surface, platforms for cell culture and other numerous applications.

\section{References}

1. Liu QG, ZhaoXS (2004) Nanoporous Materials-An Overview. Series and Chemical Engineering.

2. Zhang, Jintao, Ming Li, Chang (2012) Nanoporous metals: fabrication strategies and advanced electrochemical applications in catalysis, sensing and energy systems. Chem Soc Rev 41: 7016-7031.

3. Losic, Dusan, Santos, Abel (2010) Electrochemically Engineering Nanoporous Materials, Springer Series in Material Science 220: 1-337.

Copyright: (02016 Jibowu T. This is an open-access article distributed under the terms of the Creative Commons Attribution License, which permits unrestricted use, distribution, and reproduction in any medium, provided the original author and source are credited. 